\title{
Legalización de uniones consensuales en México*
}

\author{
Anne R. Pebley y Noreen Goldman**
}

El objetivo de este trabajo es analizar la estabilidad en el tiempo de las uniones conyugales en México y su relación con algunos factores sociodemográficos. Se utiliza la información que presenta la Encuesta Mexicana de Fecundidad de 1976, sobre los siguientes aspectos: el tipo o naturaleza de la unión al momento de su formación; la edad de la mujer a la primera unión; la presencia o no de fecundidad premarital; el grado de escolaridad de la mujer; la experiencia o inexperiencia ocupacional previa a la unión, y la condición rural-urbana que subyace al proceso de socialización de la mujer.

Entre los hallazgos más importantes del trabajo destaca que las primeras uniones urbanas presentan mayores probabilidades de terminar por divorcio o separación que las rurales y semiurbanas. A simismo, se señala la presencia de un incremento real en el tiempo de las probabilidades de disolución, lo cual lleva a la autora a plantear que en México empieza a observarse un debilitamiento de la cohesión conyugal en las uniones más jóvenes.

\section{Introducción}

La unión conyugal sancionada socialmente adquiere formas diversas en México al igual que en otros lugares de América Latina. Las formas más importantes son los matrimonios y las uniones consensuales (convivencias). Cuando se efectuó la Encuesta Mexicana de Fecundidad (EMF), aproximadamente $12 \%$ de todas las mujeres unidas lo estaban bajo el régimen de convivencia, aunque una proporción sustancialmente más importante había vivido en unión consensual en alguna época de su vida (Quilodrán, 1982). Existe amplia evidencia sobre las diferencias en la conducta de las parejas que viven en distintos tipos de unión, en donde se incluye una probabilidad de separación más elevada, y por demás importante, para las convivientes (Potter y Ojeda, 1982). Por lo tanto, en estudios sobre patrones de nupcialidad y fecundidad, es muy importante distinguir entre los distintos tipos de unión. Sin embargo, resulta complicado hacer la dis-

* Este artículo fue aceptado para publicación en el Centro de Estudios Demográficos y de Desarrollo Urbano en el primer trimestre de 1984. La terminación de Demografía y Economía, y el tiempo y prioridades involucradas en la puesta en marcha de esta nueva revista, motivaron el rezago de éste y otros importantes trabajos que apenas ahora podemos ofrecer al público de lengua española.

** Universidad de Princenton, E.U.

Las autoras agradecen a José Gómez de León y a un revisor anónimo, sus comentarios y sugerencias. 
tinción entre convivencias y matrimonios, debido a que muchas parejas que se encuentran en unión consensual deciden eventualmente contraer matrimonio civil y/o eclesiástico. Por desgracia se sabe muy poco sobre este fenómeno, al que denominaremos legalización, ${ }^{1}$ o proceso mediante el cual se legalizan las uniones consensuales.

Nuestro objetivo, en este trabajo, es examinar la legalización en México utilizando la información de la EMF que se llevó a cabo en 1976-1977 como parte de la World Fertility Survey (WFS). Este análisis es una extensión de un estudio anterior (que se describe más adelante) que trata sobre la legalización en América Latina, con base en un conjunto de encuestas que se realizaron en áreas rurales y semiurbanas durante 19691970. Éstas formaron parte de un programa de encuestas comparativas de fecundidad en América Latina (PECFAL) que condujo el Centro Latinoamericano de Demografía (Celade). Nuestro objetivo principal es describir la legalización en México como parte del patrón mexicano de nupcialidad. Examinamos las características de las mujeres que originalmente iniciaron uniones consensuales y matrimonios legales; la frecuencia y momento de legalización de las convivencias y su relación con la fecundidad, así como la estabilidad resultante de las uniones legalizadas en relación con los matrimonios legales que no tuvieron ninguna cohabitación previa. ${ }^{2}$ Nuestros resultados también se comparan con los de la encuesta mexicana PECFAL, a manera de confirmar su validez.

\section{Investigación previa}

Se ha dificultado el estudio de la legalización de las convivencias en América Latina en virtud de la insuficiencia de información. Por definición, las convivencias no están registradas en los sistemas de estadísticas vitales, y con frecuencia se encuentran subregistradas en los censos (Camisa, 1978; Flores y Goldman, 1980; Ordorica y Potter, 1980; Quilodrán, 1982). Mortara (1964) señala que usualmente sólo se registran las convivencias

\footnotetext{
1 El término "legalización" se utiliza para indicar la transición de cohabitación a matrimonio, ya sea matrimonio civil, religioso o ambos. La gran mayoría de esos matrimonios son por lo civil o una combinación de civil y religioso. Cuando se efectuó la entrevista de la EMF, únicamente $5 \%$ de las uniones existentes eran exclusivamente religiosas. Quilodrán (1982) indica que "el matrimonio 'sólo por la iglesia' ha venido en constante disminución y, actualmente, persiste sólo en las localidades más rurales".

${ }^{2}$ La legalización de convivencias no es la única forma de transición en uniones con la misma pareja. El análisis exhaustivo de este tema debería incluir también el examen de lo que podríamos llamar santificación de matrimonios, es decir, el cambio de un matrimonio civil a uno religioso santificado en una segunda ceremonia por algún sacerdote un poco después del matrimonio civil. Por desgracia, la información de la EMF y la información previa de las distintas encuestas PECFAL no nos permiten determinar si un matrimonio reportado retrospectivamente como civil y religioso se inició como tal o si el rito religioso fue después de la ceremonia civil.
} 
que "tienen un cierto carácter o permanencia" (pp. 73-74); que las mujeres reportan más que los hombres la existencia de estas uniones, y que es probable que algunos encuestados reporten que están casados legalmente antes de admitir que se encuentran bajo el régimen de unión libre. El análisis de la legalización se complica aún más debido a que en la mayoría de los sistemas de estadísticas vitales y los censos no se distingue entre matrimonios legales con/sin cohabitación previa. La información del sistema de estadísticas vitales de Panamá (1967) y Guatemala (1957) es una excepción, porque nos indica que cerca de 30 y 15 por ciento, respectivamente, del primer matrimonio legal estuvo precedido por cohabitación con la misma pareja (Camisa, 1978).

Sólo dos series de encuestas sobre fecundidad de las que se han llevado a cabo en América Latina durante los últimos veinte años, incluyeron una historia completa de uniones: las encuestas rurales PECFAL que se llevaron a cabo en 1969 y 1970 en varios países, y las encuestas nacionales de fecundidad que se realizaron desde 1975 como parte del WFS. Las encuestas PECFAL intentaron reunir un amplio margen de información detallada sobre fecundidad y nupcialidad. La historia de uniones registra los cambios en los tipos de unión como uniones separadas, y considera la legalización como una de las posibles maneras de finalizar la unión. La EMF de 1976 empleó un formato similar para la historia de uniones, si bien un poco más detallado. En contraste, las demás encuestas en América Latina, que formaron parte del WFS, utilizaron una versión modificada de su cuestionario regular, lo que produjo fallas para determinar si una mujer ha tenido más de un tipo de unión con la misma pareja. Esto porque la terminación de una convivencia y el comienzo de un matrimonio legal con la misma pareja se registraron como unión única. Esta limitación del cuestionario del WFS no sólo hace que el estudio de la legalización en todos los países sea irrealizable, con excepción de México, sino que confunde también el análisis de la disolución de la unión en un grado desconocido (Goldman, 1981).

Los dos estudios previos que examinaron la legalización en América Latina utilizaron la información proveniente de las encuestas PECFAL en México, Colombia, Costa Rica y Perú. Las encuestas PECFAL se basaron en muestras autoponderadas de dos mil a tres mil mujeres, en edades de 15 a 49 años, que vivían en áreas rurales o semiurbanas, mismas que el Celade define como áreas con una población de menos de 20000 habitantes. Casi $70 \%$ del total de la población de cada uno de estos países vivía en áreas de ese tamaño en la época en que se efectuó la encuesta. Rosero (1978) calculó indirectamente la frecuencia de la legalización para cada país en el primer estudio. Mencionó que $10.2 \%$ de las mujeres mexicanas con edades de 15 a 49 años había tenido tanto convivencias como matrimonios legales, y que $90.3 \%$ de estas mujeres tuvo una primera unión bajo forma de convivencia. Las cifras comparables para los otros tres países 
van de 8.1 al 15.2 por ciento, y de 79.6 a 97.7 por ciento, respectivamente. También construyó tablas de vida por duración de unión para convivencias y para matrimonios legales. Sin embargo, como la terminación de las uniones puede deberse a legalización, separación y viudez combinadas, no es posible derivar de esta información la frecuencia de las legalizaciones ${ }^{3}$ Rosero, al igual que otros autores (CEPAL, 1975; Camisa, 1978; Stycos, 1968), conjeturaba que un gran número de legalizaciones se llevan a cabo como resultado de un embarazo o nacimiento, aunque no hizo el intento de verificar esta hipótesis.

Goldman y Pebley (1981) utilizan en un segundo análisis la información de la encuesta PECFAL, así como el método de tablas de vida, a fin de observar más directamente la frecuencia de la legalización por duración de la unión. Los resultados indicaron que la proporción de convivencias que fueron legalizadas dentro de los primeros diez años de cohabitación, era de $22 \%$ en México, $19 \%$ en Colombia, $18 \%$ en Costa Rica y significativamente más elevada en Perú (35\%), en donde también era más común la convivencia. En los cuatro países, sin importar la duración de la unión, era más probable que las mujeres que iniciaron su primera unión libre después de los 17 años y que tenían algún grado de escolaridad, terminaron legalizando tal unión. Las mujeres en México clasificadas como convivientes y católicas, también tenían más probabilidades, comparadas con las demás mujeres, de legalizar su unión. La probabilidad de que se llevara a cabo un matrimonio legal dentro de los diez años posteriores al comienzo de la convivencia en este país, era de $38 \%$ para las católicas practicantes, en comparación con $22 \%$ para otras mujeres. Sin embargo, en los tres países restantes la religiosidad no representaba una gran diferencia. Los resultados también indicaron que el momento de la legalización no parecía estar relacionado con el primer embarazo o nacimiento. De hecho, no existía ninguna diferencia en la probabilidad de legalización entre las parejas que tenían hijos y las que no los tenían al estar en convivencia.

\section{Metodología}

En este análisis medimos el riesgo de legalización con tablas de vida. Las tablas de vida que están clasificadas por duración de la unión (por ejemplo, meses o años desde el inicio de la convivencia) toman en cuenta directamente el tiempo de exposición al riesgo de legalización. De esta for-

\footnotetext{
${ }^{3}$ Más aún, en las tablas de vida de Rosero las mujeres que habían formado una segunda unión, o uniones de orden subsiguiente, entran nuevamente en la tabla de vida en su duración respectiva desde el inicio de su primera unión; de ahí que no sea posible inferir de estas tablas ni siquiera la frecuencia de finalización de las uniones.
} 
ma, arrojan más información que las medidas simples del porcentaje de convivencias que se legalizaron por fecha de entrevista. $O$, más específicamente, las tablas de vida incorporan la información de ambos tipos de unión, las que han terminado y las que permanecieron intactas en la fecha de la entrevista, al determinar el estado de una unión en cada duración.

El cálculo de las tablas de vida puede basarse en tablas de decremento, tanto doble como simple. En las tablas de decremento doble se consideran dos tipos de eventos que pueden dar fin a una convivencia: 1) legalización y 2) cualquier otro tipo de evento, por ejemplo, separación o muerte de alguna de las partes integrantes. Si bien es cierto que la legalización es ei interés principal, también es cierto que la frecuencia de otros tipos de término de la unión pueden afectar el porcentaje de las convivencias que realmente se legalizan. Esto es, si la mayoría de las convivencias terminan en una separación, entonces serían muy pocas las que podrían terminar en legalización. Por el contrario, en las tablas de vida de decremento simple, se registra la legalización como única forma de término de la unión, de ahí que la separación y muerte de alguna de las partes queden eliminadas. Las convivencias que se disuelven por esias últimas causas contribuyen a la exposición al riesgo de legalización hasta el momento de la separación o la muerte. De ahí que las probabilidades de las tablas de vida de decremento simple sean hipotéticas, ya que se refieren al porcentaje de convivencias que podrían legalizarse por duraciones sucesivas si no se terminaran por otras causas.

La construcción de tablas de vida de decremento doble abarca los cálculos $D_{1 i}$ y $D_{2 i}$, del número de convivencias que terminan por causa de legalización, separación o muerte de una de las partes durante el intervalo $(i, i+1)$, y un cálculo $N_{i}$, del número de convivencias que están intactas al comienzo del intervalo. Las probabilidades de legalización específicas por duración de la unión $\left(q_{1 i}\right)$, y por separación o viudez $\left(q_{2 i}\right)$ son la relación entre los eventos y las uniones existentes:

$$
\begin{gathered}
q_{1 i}=D_{1 i} / N_{i}, \\
y \\
q_{2 i}=D_{2 i} / N_{i}^{4}
\end{gathered}
$$

La probabilidad acumulada de que una convivencia termine en la duración $i$ como resultado de una sola causa involucra la suma de las probabilidades de que finaiice por esa causa en cada duración anterior a la i. En símbolos, la probabilidad acumulada de que se legalice en la duración, $i, Q_{1 i}$, es igual a

${ }^{4}$ De hecho, los cálculos son un poco más complicados. La mitad de las convivencias de las mujeres que fueron entrevistadas durante el intervalo $(i, i+1)$ se omiten del denominador $N_{i}$. 


$$
Q_{1 i}=\prod_{j=0}^{i-1} P_{j} q_{1 j^{\prime}}
$$

en donde $p_{j}$ es la probabilidad de que quede intacta en la duración $j$,

$$
P_{j}=\prod_{z=0}^{j-1}\left(1-q_{1 z}-q_{2 z}\right)
$$

En virtud de que $p_{i}$ incluye los valores de $q_{2 i}$ debe resaltarse que la probabilidad acumulada de legalización en una tabla de decremento doble depende del riesgo de separación y muerte de una de las partes. La probabi- lidad acumulada que termine la convivencia en la duración $i$, como resultado de cualquier causa, es simplemente $Q_{i}=Q_{1 i}+Q_{2 i}$.

La construcción de una tabla de vida de decremento simple para legalizaciones incluye el cálculo, $D_{i}$, del número de convivencias que se legalizan durante el intervalo $(i, i+1)$, y el cálculo, $N_{i}$, del número de convivencias que se encuentran intactas al comienzo del intervalo. Las probabilidades específicas por duración $q_{i}=D_{i} / N_{i}$, son casi idénticas a los valores de $q_{1 i}$ de la tabla de decremento doble ${ }^{5}$ pero la probabilidad acumulada, $Z_{i}$, de legalización por duración $i$,

$$
Z_{i}=1 \Pi_{j=o}^{i-1}\left(1-q_{j}\right)
$$

será siempre cero, o mayor que el valor correspondiente al decremento doble $Q_{1 i}$ puesto que no se considera la separación o muerte como término de la unión. Hacemos hincapié en que los valores de $Z_{i}$ corresponden a los valores $\left(1-\ell_{i}\right)$ en una tabla de vida estándar.

Las probabilidades específicas por duración $q_{i}$ son bastante irregulares en virtud de que el tamaño de la muestra es pequeño en cada intervalo de estas tablas de vida. De ahí que en la mayoría de los cuadros presentamos probabilidades acumuladas. Aunque los valores de decremenio doble $\left(Q_{1 i}\right)$ son indicadores más fidedignos de los porcentajes reales de las convivencias que se legalizan, en comparación con los valores de las tablas de decremento simple $\left(Z_{i}\right)$, la comparación de $Q_{1 i}$ a través de subgrupos puede ofrecer un panorama distorsionado por incidencias diferenciales de separación o viudez. Puede suceder, por ejemplo, que dos subgrupos tengan idénticas probabilidades de legalización específicas por duración $\left(q_{1 i}\right)$, pero distintos valores $Q_{1 i}$ a causa de tasas de separación distintas: para $q_{1 i}$ fija, mientras mayor probabilidades de separación $q_{2 i}$ menor la $Q_{1 i}$.

Consideramos inicialmente las probabilidades de las tablas de decremento simple y doble, para la muestra total de convivencias, pero única-

${ }^{5}$ En la tabla de decremento simple se omite del denominador $N_{i}$ a la mitad de las convivencias de las mujeres entrevistadas, separadas o viudas, durante el intervalo (i, $i+1)$. De ahí que el valor de $q_{i}$ en la tabla de vida de decremento simple puede ser un poco más elevado que el valor de $q_{l i}$ en la tabla de decremento doble. 
mente utilizamos valores de decremento simple $\left(Z_{i}\right)$ para efectuar las comparaciones entre los subgrupos. ${ }^{6}$ El lector no debe olvidar que los valores $Z_{i}$ son hipotéticos, ya que representan la proporción de convivencias que podrían legalizarse por duraciones sucesivas, en caso de que ninguna unión terminara en muerte o separación de alguna de las partes. Sin embargo, las tablas de decremento simple arrojan, al eliminar todos los riesgos en competencia, una comparación estandarizada de los riesgos de legalización entre los distintos grupos de mujeres.

\section{Información}

La información que se utilizó en este análisis proviene de la EMF 19761977. La muestra de la EMF es autoponderada; abarcó 13080 hogares e intentó ser representativa de la población nacional. Al igual que en otros casos de la WFS se utilizaron cuestionarios breves a ser llenados en cada hogar y de ahí se seleccionaron a las mujeres adecuadas que posteriormente fueron entrevistadas utilizando un cuestionario individual y detallado. Las mujeres eran elegibles para la entrevista si tenían entre 20 y 49 años de edad, o bien si tenían de 15 a 19 y habían estado alguna vez casadas o tenían hijos (EMF, Primer informe nacional, 1979, vol. 1, p. 51). La información que se utiliza en este estudio, de un total de 7310 mujeres, se deriva exclusivamente de las entrevistas individuales y en especial de las historias de unión y de nacimientos. En la historia de unión se pidió a las mujeres que proporcionaran el año y mes del inicio de cada unión, tipo de unión (civil, religiosa o convivencia), año y mes de disolución (en caso conducente), y la razón de disolución (incluyendo si era unión legalizada o no). En la historia reproductiva de la EMF se recogió información para cada embarazo en cuanto a fecha de término, tipo de resultadc (nacimiento de niños vivos, mortalidad fetal, etc.), y tiempo de gesta. ción para embarazos que no resultaron en nacimientos vivos.

Ordorica y Potter (1980) evaluaron la calidad de la información de la EMF utilizando pruebas de consistencia interna y comparaciones con otras fuentes de información. Concluyeron que, en general, "todo indica que la encuesta se llevó a cabo de manera cuidadosa, y los resultados en conjunto parecen estar libres de error de respuesta en una medida razona-

\footnotetext{
${ }^{6}$ No obstante, el cálculo de las tasas de decremento simple requiere del supuesto de que los dos eventos de interés se realicen independientemente uno del otro. Este supuesto puede no ser válido para el caso de legalización y separación. Por desgracia tampoco resulta convincente, como se comenta en el texto, el uso de las tasas de legalización de decremento doble, porque no pueden compararse a través de subgrupos en virtud de las diferentes tasas de separación. Al habernos enfrentado a dos alternativas que definitivamente no son las ideales, hemos preferido presentar las tasas de decremento simple a fin de facilitar la interpretación de nuestros resultados. De cualquier modo, la dirección de los mismos permanece igual para ambas tasas de decremento, simple y doble.
} 
ble" (p. 28). Más específicamente, sus resultados indican que "la información sobre nupcialidad de la EMF está significativamente menos afectada por error de respuesta que otras encuestas en la serie de la WFS que se han llevado a cabo en países con un nivel de desarrollo económico similar, si bien existe cierta evidencia de error en la ubicación de los nacimientos para las cohortes más viejas"' (p. 28).

\section{Comparación de la información de la EMF con la de la encuesta PECFAL}

Con el fin de comprobar adicionalmente la calidad de la información y de facilitar la comparación posterior de resultados entre las encuestas EMF y PECFAL para México, analizamos la información sobre nupcialidad y fecundidad en los dos casos en lo que respecta al periodo de tiempo y a la población que cubren ambas encuestas. Resulta relativamente fácil derivar tabulaciones de la EMF que puedan compararse con los resultados de la PECFAL con respecto al periodo de tiempo: se consideran únicamente los eventos anteriores al 1 de enero de 1970 (aproximadamente el punto medio de las entrevistas de la PECFAL), y la edad para las mujeres que se entrevistaron en la EMF puede calcularse para esta fecha. Como se ilustra en el diagrama de Lexis de la figura 1, con el fin de obtener muestras similares respecto de la edad, se eliminaron de la EMF a las mujeres de menos de 22 años y de la PECFAL a las de 43 años o más. Esto se debió a que las mujeres de menos de 22 años de la EMF no llenaban el criterio de edad mínima de la PECFAL, o sea, contar con 15 años en 1970. Igualmente, las mujeres de 43 años (cumplidos), en la PECFAL tendrían 50 años o más en la época de la EMF, con lo que quedarían excluidas de su muestra. El resultado de los cálculos a enero de 1970 se basa entonces en mujeres con edades de 15 a 42 años, ya sea para estimaciones derivadas de la EMF o de la encuesta PECFAL.

Sin embargo, el total de la muestra pre-1970 de la EMF no puede compararse de manera estricta con la muestra de la encuesta PECFAL en virtud de que todas estas últimas entrevistadas tenían que vivir en áreas de menos de 20000 habitantes, siguiendo el criterio de elegibilidad. Es difícil determinar la selección de una submuestra de la EMF que pueda compararse a los requerimientos de residencia rural de la encuesta PECFAL. En virtud de que la EMF recopiló la historia migratoria de cada entrevistada, teóricamente sería posible restringir las tabulaciones de la EMF sólo para las mujeres que en 1970 vivían en poblaciones de menos de 20000 habitantes. Sin embargo, no se codificó la información necesaria en cintas. Escogimos un enfoque más simple, aunque menos satisfactorio. Comparamos a las entrevistadas PECFAL con dos grupos: el total de la muestra de la EMF y la muestra de mujeres que vivían en localidades de menos 
FIGURA 1

Diagrama de Lexis que rauestra las edades de la encuesta EMF en el momento de la encuesta PECFAL

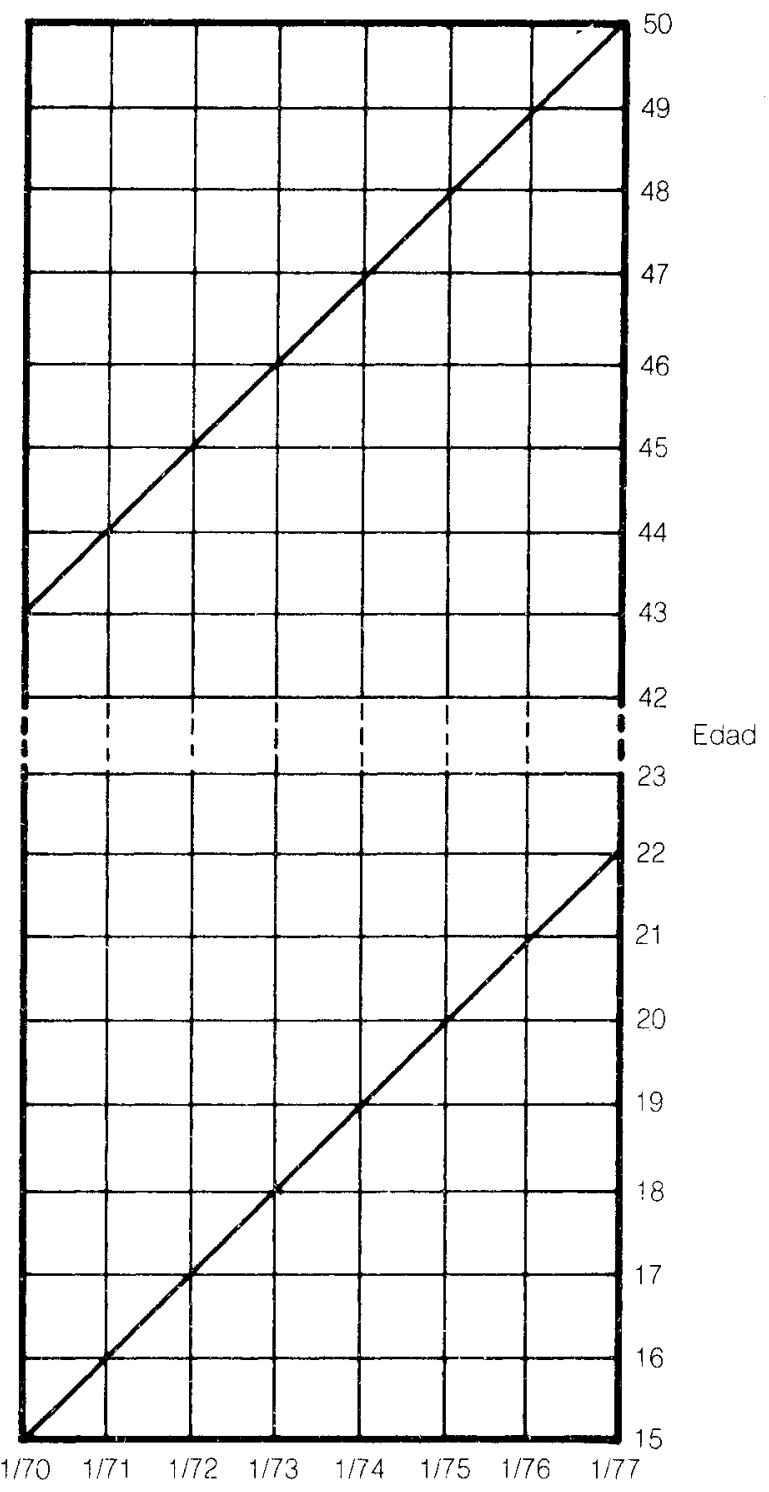


de 20000 habitantes en la fecha de la EMF. Existen dos razones por las que esta segunda muestra no sería comparable con la muestra rural de PECFAL; la migración que se ha efectuado primordialmente de áreas rurales a áreas urbanas entre 1969 y 1976, y el crecimiento de los lugares de menos de 20000 habitantes a más de $20000 .{ }^{7}$ Deben tomarse en cuenta estas limitaciones en el momento de hacer las interpretaciones.

En el cuadro 1 se compara la distribución por edades, la proporción de primeras uniones que son convivencias, la proporción de mujeres unidas actualmente, y el número promedio de hijos nacidos vivos de las mujeres entrevistadas en la encuesta PECFAL entre 1969 y 1970, con los valores correspondientes para el total de la encuesta y para la muestra rural de la EMF en enero de 1970. Los grupos de edades en el lado izquierdo reflejan la edad en el momento de la entrevista para la encuesta PECFAL, y la edad en enero de 1970 para las dos muestras de la EMF. En este cuadro no se incluyen los grupos quinquenales de edad 40 a 44 y 45 a 49 , porque las mujeres más viejas que podrían entrar en la EMF no habrían alcanzado la edad de 43 años en 1970.

La distribución total y rural por edades para las muestras de la EMF se asemeja considerablemente a la de la encuesta PECFAL. La proporción de mujeres que estaban casadas (legalmente o en convivencia) en el total de la muestra de la EMF es muy similar a la de la encuesta PECFAL para cada uno de los grupos de edad. Ninguna de las diferencias, exceptuando al grupo de 20 a 24 años, son superiores a $1 \%$. La proporción que estaba casada en la muestra rural de la EMF es muy diferente a la total de la EMF y a la de la encuesta PECFAL, lo que sugiere que la emigración de áreas de menos de 20000 habitantes pudo haber sido selectiva para mujeres que eran menos susceptibles de casarse en cualquier edad.

Sin embargo, el tercer panel del cuadro 1 muestra que las entrevistadas rurales de la EMF se acercan más a las entrevistadas de la encuesta PECFAL en términos del tipo de unión que tenían al final de 1969. La proporción de primeras uniones que eran convivencias para la muestra PECFAL, a excepción de las edades de 15 a 19 años, se asemeja más a la muestra rural de la EMF que a las cifras totales. Resultan muy semejantes en conjunto las cifras de las muestras rural de la EMF y PECFAL, si bien es algo inquietante la diferencia en las cifras para los grupos de 15 a 19 y de 20 a 24 años.

El último panel del cuadro 1 muestra el número promedio de niños nacidos vivos (en la entrevista o en enero de 1970) para las tres muestras. Los promedios de los dos primeros grupos de edad de la encuesta son muy semejantes a los del total de la muestra de la EMF, mientras que los pro-

\footnotetext{
${ }^{7}$ Independientemente del procedimiento, un tercer problema al que nos enfrentamos es el de la mortalidad durante el periodo en cuestión. Sin embargo, suponemos que la mortalidad entre las mujeres en edad reproductiva en México es bastante baja.
} 


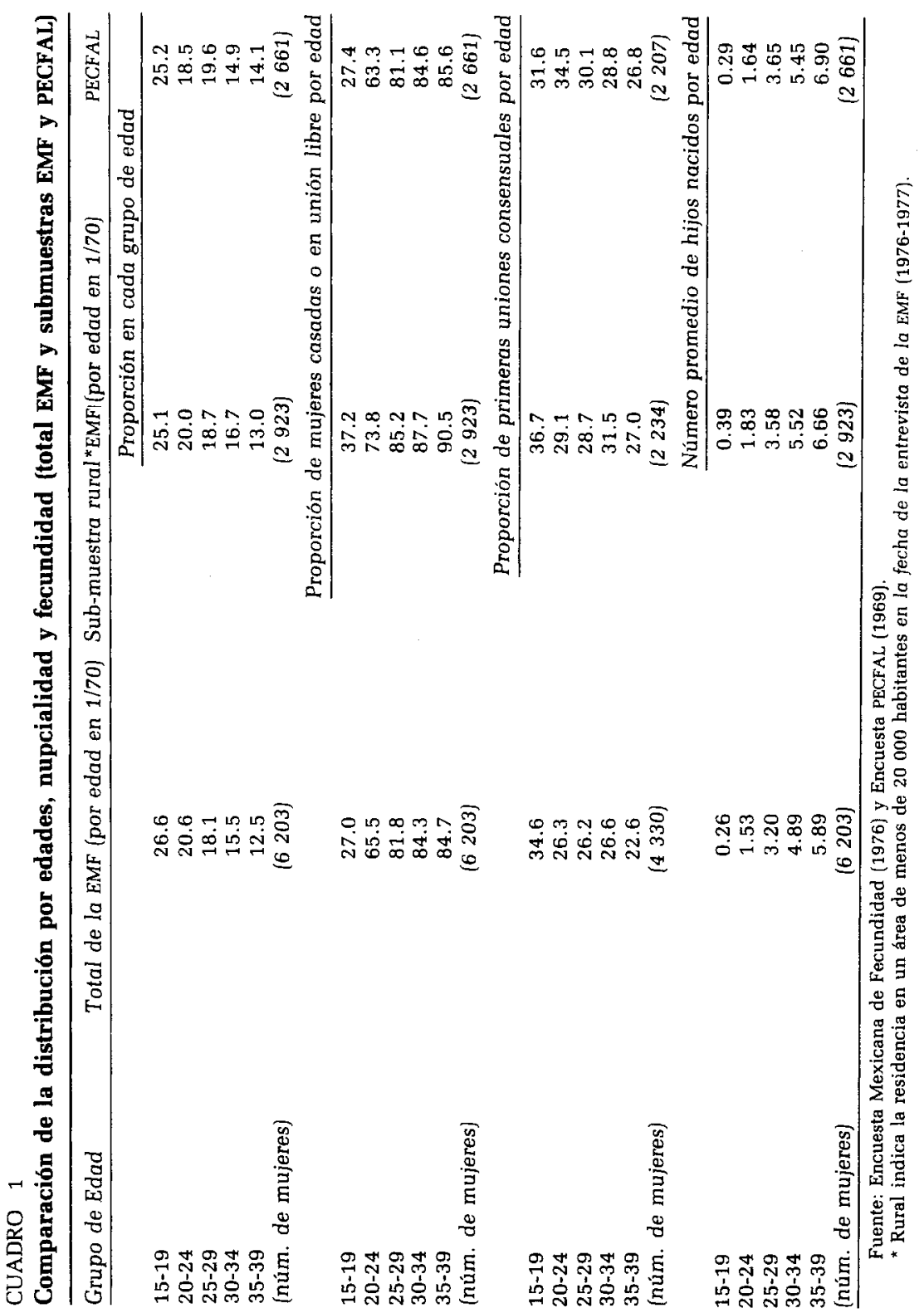


medios de la PECFAL para las tres cohortes de más edad se asemej. a los de la muestra rural de la EMF. La explicación puede ser que exist... más probabilidades de que las mujeres más jóvenes abandonasen las áreas rurales. Esto es, que era probable que las mujeres de 25 años o más que vivían en un área rural en 1969 fueran el mismo grupo de mujeres que se encontraba en áreas rurales en 1976. Los valores más bajos en la muestra de la EMF para los grupos rurales de mayor edad puede ser un signo de omisión de los nacimientos por parte de las mujeres más viejas, en virtud de que las entrevistadas de la EMF reportaron los mismos eventos con una duración más prolongada desde su ocurrencia que las entrevistadas correspondientes del PECFAL.

En resumen, a pesar de la falta de comparabilidad geográfica precisa, los resultados de las encuestas PECFAL y EMF concuerdan razonablemente. En la mayor parte de los casos, los resultados PECFAL se ubican entre los correspondientes al total de la EMF y a la submuestra rural, como podríamos esperar de los patrones recientes de migración y el crecimiento de las áreas rurales.

\section{Resultados}

\section{Frecuencia de convivencias}

Nuestras estimaciones se restringen a las uniones libres porque nuestro análisis privilegia las probabilidades de legalización de dichas uniones. Sin embargo, antes de examinar la frecuencia de legalización consideramos someramente la ocurrencia de convivencias en la muestra de la EMF. El cuadro 2 muestra el porcentaje de uniones que son convivencias registradas en la historia correspondiente de la EMF con respecto a distintas características de las entrevistadas. Correspondía a convivencias $28 \%$ del total de uniones conyugales. Las primeras uniones, que incluyen casi el total de uniones, son más frecuentemente convivencias en el área rural, ${ }^{8}$ que en las áreas urbanas. Para las segundas o subsiguientes uniones, las convivencias son ligeramente más comunes en las áreas urbanas. En total, la frecuencia de uniones libres es mayor entre las uniones de orden superior, si bien las diferencias se borran en el caso de las áreas rurales.

La proporción de uniones libres es de 31.7 para la muestra PECFAL, cifra muy cercana al $31.2 \%$ de la muestra rural de la EMF. Las cifras de la muestra rural de la EMF y las de la encuesta PECFAL para primeras uniones también concuerdan razonablemente. Sin embargo, mientras que

${ }^{8}$ A menos que se indique lo contrario, en este trabajo se adoptó la definición de la encuesta PECFAL para el término rural: localidades con menos de 20000 habitantes en la fecha de la entrevista. 
$41.9 \%$ de las uniones de orden superior en la encuesta PECFAL son convivencias, la cifra comparable para la muestra rural de la EMF es únicamente de $30 \%$.

CUADRO 2

Porcentaje de las uniones que son consensuales

\begin{tabular}{|c|c|c|c|c|c|c|}
\hline \multirow[b]{2}{*}{ Total de uniones } & \multicolumn{2}{|c|}{$\begin{array}{c}\text { Tolal de la } \\
\text { muestra }\end{array}$} & \multicolumn{2}{|c|}{ Urbana } & \multicolumn{2}{|c|}{ Rural } \\
\hline & 28.0 & $(7602)$ & 24.6 & $(3716)$ & 31.2 & (3 889) \\
\hline Primeras uniones & 27.3 & (6 253) & 23.1 & $(3098)$ & 31.5 & (3 155) \\
\hline Segunda y subsiguientes & 31.1 & (1 349$)$ & 32.5 & $(615)$ & 30.0 & (734) \\
\hline \multicolumn{7}{|l|}{ De las primeras uniones } \\
\hline \multicolumn{7}{|l|}{ Edad a la entrevista } \\
\hline$<20$ & 40.9 & $(470)$ & 38.7 & $(173)$ & 42.1 & ( 297) \\
\hline $20-29$ & 27.0 & (2 322) & 22.3 & (1 179) & 31.9 & (1 143) \\
\hline $30-39$ & 26.4 & (2 041) & 23.3 & $\left(\begin{array}{ll}1 & 030\end{array}\right)$ & 29.5 & (1 011$)$ \\
\hline $40-49$ & 24.6 & $(1420)$ & 20.3 & $(716)$ & 29.1 & (704) \\
\hline \multicolumn{7}{|l|}{ Edad a la unión } \\
\hline$\leqslant 17$ & 34,1 & (2 919) & 31.2 & (1 159) & 36.0 & $\left(\begin{array}{ll}1 & 760\end{array}\right)$ \\
\hline$>17$ & 21.4 & (3 334) & 18.2 & $\left(\begin{array}{ll}1 & 939\end{array}\right)$ & 25.7 & (1395) \\
\hline \multicolumn{7}{|l|}{ Escolaridad } \\
\hline Ninguna & 40.0 & $\left(\begin{array}{ll}1 & 404\end{array}\right)$ & 35.8 & $(400)$ & 41.6 & $(1004)$ \\
\hline Alguna & 23.7 & $(4849)$ & 21.2 & $(2698)$ & 26.7 & $\left(\begin{array}{ll}2 & 151\end{array}\right)$ \\
\hline Primaria & 26.9 & (3 799) & 26.7 & $(1786)$ & 27.1 & $\left(\begin{array}{ll}2 & 013\end{array}\right)$ \\
\hline Secundaria y más & 11.9 & $\left(\begin{array}{ll}1 & 050\end{array}\right)$ & 10.4 & ( 912) & 21.7 & ( 138$)$ \\
\hline
\end{tabular}

Fuente: Encuesta Mexicana de Fecundidad (1976).

La segunda parte del cuadro 2 indica que es más probable que la primera unión de una mujer sea consensual si era más joven en la fecha de la entrevista, si era más joven al momento de su unión, y si tenía poca o ninguna escolaridad. Estos últimos dos resultados son consistentes con los hallazgos de análisis previos en el sentido de que las mujeres constituyen uniones libres a edades más tempranas que los matrimonios legales, y que las mujeres en unión libre corresponden, por lo general, a un estrato social más bajo (Yaukey y Thorsen, 1972; Michielutte, et al., 1973). La proporción de uniones libres en la muestra rural de la EMF clasificada por edad en el momento de la entrevista, edad cuando se realizó la unión, así como escolaridad, es muy similar a la de la encuesta PECFAL (Goldman y Pebley, 1981) 


\section{Frecuencia de legalización}

Las cifras del cuadro 3 sugieren que las convivencias se convierten con frecuencia en matrimonios legales, a pesar de que suman más de la cuarta parte del total de uniones en México y se consideran como una alternativa aceptable al matrimonio civil o religioso. La EMF registró que aproximadamente la mitad de las uniones libres se convirtieron en matrimonios al alcanzar la duración de 25 años. Muy pocas convivencias permanecen

\section{CUADRO 3}

Proporción de las convivencias que terminan por diferentes causas en las tablas de decremento doble, y proporción de convivencias legalizadas en la tabla de decremento simple

\begin{tabular}{|c|c|c|c|c|}
\hline \multirow{2}{*}{$\begin{array}{l}\text { Duración de } \\
\text { la unión } \\
\text { (años) }\end{array}$} & \multicolumn{3}{|c|}{ Decremento doble } & \multirow{2}{*}{$\begin{array}{l}\text { Decremento } \\
\text { simple } \\
\text { Legalización }\end{array}$} \\
\hline & Legalización & $\begin{array}{c}\text { Separación } \\
\text { o viudez }\end{array}$ & $\begin{array}{l}\text { Todo tipo } \\
\text { de causa }\end{array}$ & \\
\hline 1 & .18 & .06 & .24 & .17 \\
\hline 2 & .21 & .09 & .30 & .22 \\
\hline 3 & .25 & .12 & .37 & .25 \\
\hline 5 & .29 & .17 & .46 & .31 \\
\hline 10 & .37 & .23 & .60 & .41 \\
\hline 15 & .42 & .27 & .69 & .49 \\
\hline 20 & .47 & .30 & .77 & .58 \\
\hline 25 & .52 & .32 & .84 & .68 \\
\hline
\end{tabular}

Rural $(N=915)$

\begin{tabular}{rllll}
1 & .18 & .05 & .23 & .17 \\
2 & .21 & .08 & .29 & .22 \\
3 & .24 & .11 & .35 & .25 \\
5 & .28 & .15 & .43 & .30 \\
10 & .35 & .21 & .56 & .38 \\
15 & .41 & .25 & .66 & .48 \\
20 & .45 & .27 & .72 & .54 \\
25 & .52 & .29 & .81 & .65 \\
& & & & \\
& & & Urbana $(\mathrm{N}=1213)$ & \\
1 & .18 & .06 & .24 & .17 \\
2 & .21 & .11 & .32 & .22 \\
3 & .25 & .15 & .39 & .26 \\
5 & .30 & .18 & .48 & .32 \\
10 & .39 & .25 & .65 & .45 \\
15 & .43 & .30 & .73 & .52 \\
20 & .49 & .35 & .84 & .65 \\
25 & .52 & .37 & .89 & .72 \\
\hline
\end{tabular}

Fuente: Encuesta Mexicana de Fecundidad (1976) 
como tales después de este periodo, en virtud de los efectos combinados de legalización, separación o viudez. Tal como lo indican las tablas de decremento doble, la frecuencia de legalización es casi la misma en áreas urbanas y rurales. No obstante, es más probable que las mujeres de las áreas urbanas finalicen su convivencia en separación, lo que da por resultado una tasa de terminación más elevada en las áreas urbanas debido a todas las causas. Tanto las legalizaciones como las separaciones tienden a efectuarse al inicio de la unión; $30 \%$ de las uniones finalizan en los dos primeros años. Los valores de las tablas de decremento simple indican que dos terceras partes de las convivencias se convierten eventualmente en matrimonios legales, en ausencia de separación o viudez; las tasas de legalización de decremento simple en las áreas urbanas son ligeramente más elevadas que en las áreas rurales.

Las probabilidades de legalización que se muestran en el cuadro 3 son sustancialmente más elevadas que las que encontramos en la encuesta PECFAL (cuadro 4), aun cuando sólo se consideren las cifras de la muestra rural de la EMF. Tales diferencias podrían ser el resultado de un buen número de factores, que incluyen un cambio en el patrón de legalización después de 1970, errores de registro en las dos encuestas, y diferencias en los marcos muestrales de las encuestas PECFAL y EMF. En el cuadro 4 se calcularon nuevamente las probabilidades de legalización de la EMF, restringiendo la muestra a uniones que empezaron antes de enero de 1970,

CUADRO 4

Probabilidad de decremento simple de legalizar una convivencia por duración de la unión (total PECFAL y submuestras PECFAL y EMF)

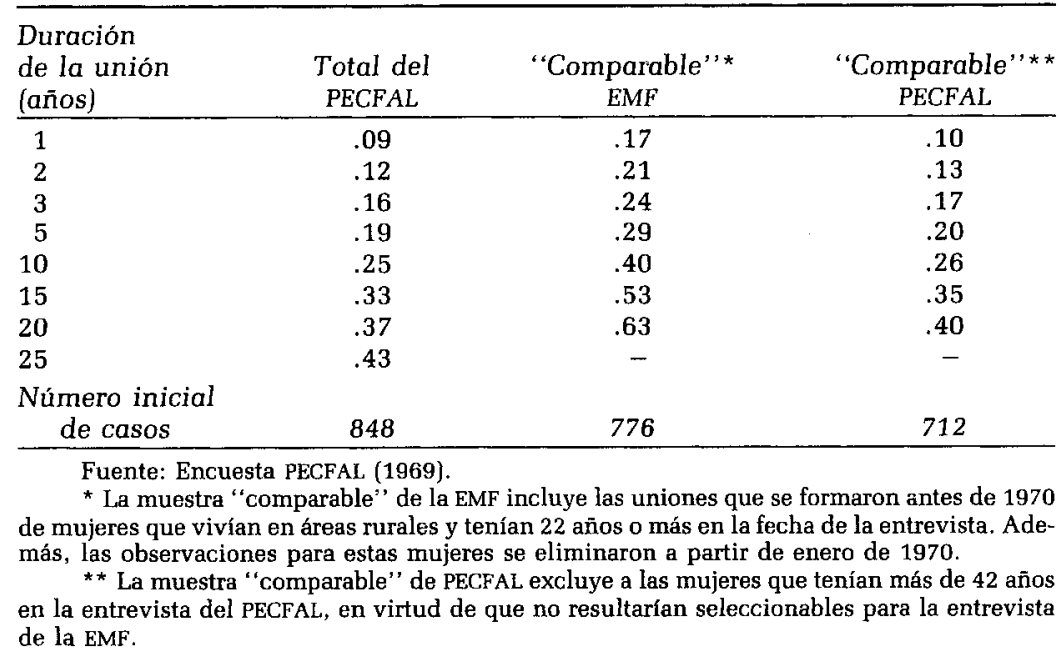


lo cual involucra a mujeres que vivían en áreas rurales y que tenían más de 22 años en la fecha de la entrevista de la EMF (es decir, por lo menos 15 años en enero de 1970). Además, prescindimos de todas las uniones que empezaron en enero de 1970 a fin de igualar el efecto respectivo en la entrevista de la PECFAL. A fin de asegurar la comparabilidad, se calcularon nuevamente las probabilidades de la encuesta PECFAL con el objeto de eliminar a las mujeres de mayor edad ( 43 años y más) que no habrían sido elegibles siete años más tarde en la entrevista de la EMF. Las cifras del cuadro 4 manifiestan que las probabilidades acumuladas de legalización para la muestra PECFAL permanecen más bajas que las de la EMF, aun cuando se utilizan las muestras comparables. En la figura 2 se muestran las probabilidades de legalización específicas por duración (no acumuladas) para las muestras comparables de la encuesta PECFAL y la EMF, para los primeros diez años de unión. Esta figura indica que la mayoría de las diferencias en las tasas de legalización provienen de la primera duración. Ambas encuestas determinaron que las tasas de legalización bajan drásticamente después del primer año y de ahí en adelante se mantienen relativamente constantes. Si se restringe la comparación a la primera unión de las dos muestras, el patrón resulta en esencia el mismo.

FIGURA 2

México: Probabilidades de legalización específicas por duración (muestras comparables de las encuestas EMF y PECFAL)

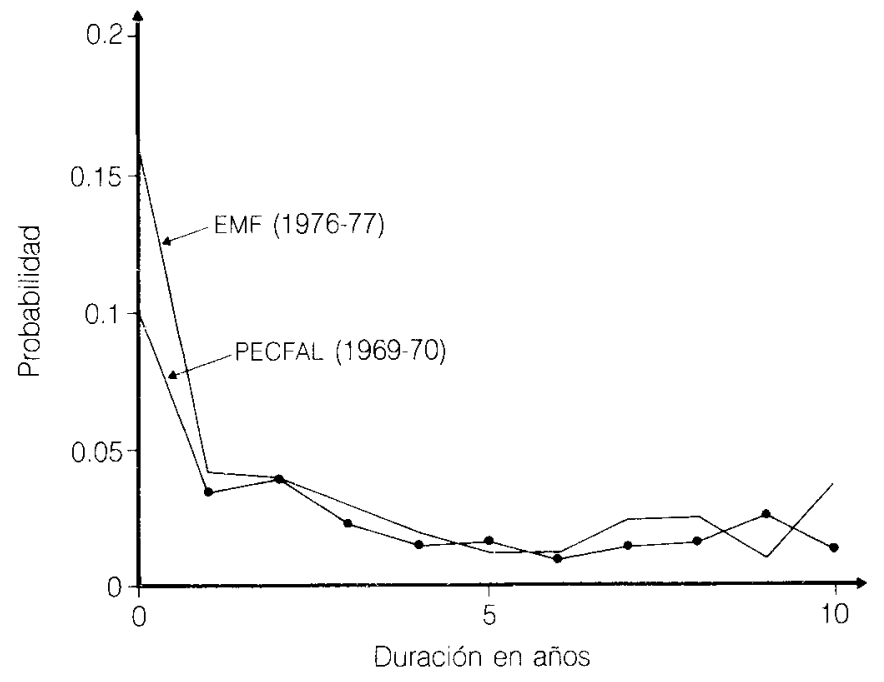


Se calcularon las probabilidades de legalización de la EMF para uniones que se efectuaron antes de 1970 en áreas rurales pequeñas (que en 1976 contaban con menos de 2500 habitantes), a fin de determinar si la diferencia en las probabilidades de legalización se debe a la falta de comparabilidad geográfica entre las muestras. Sin embargo, la tasa de legalización de cada duración para esta submuestra varía muy poco respecto al total de la muestra de la EMF. Por tanto, no parece factible que la falta de comparabilidad geográfica sea la explicación.

Una comparación entre las historias de uniones de la EMF y la encuesta PECFAL sugiere que parte de las diferencias existentes en las tasas de legalización observadas pueden ser causadas por el distinto diseño del cuestionario. En el cuestionario de la EMF se preguntabá a la entrevistada sobre cada matrimonio, la fecha en que éste comenzó, si tuvo cohabitación previa con el mismo hombre y, si fue así, la fecha en que comenzó a vivir con su pareja. Además, el formato tabular en el que se registran las uniones previas aclara que cada unión (con el mismo hombre o no) se registra por separado, y que la legalización es una forma adecuada de finalizar la unión.

A pesar de que el intento era similar en el cuestionario de la encuesta PECFAL, las preguntas están organizadas en una forma más confusa. Se preguntó a las entrevistadas en el caso de cada matrimonio si habían vivido con el mismo hombre antes de efectuarse el mismo. Sin importar la respuesta, después se le pregunta "¿en qué año y mes se casó (unió) usted?"'. Se deja al criterio del entrevistador que registre la unión que precede el matrimonio como unión separada con fecha de iniciación separada. Esta secuencia de preguntas también pudo haber originado cierta confusión entre las entrevistadas en relación a si deberían dar la fecha del matrimonio (la legalización) o la fecha en que empezaron a cohabitar. Más aún, se preguntć a las entrevistadas en el caso de las uniones que habían terminado: “¿Cómo se terminó este matrimonio (convivencia): muerte, separación o divorcio?", aun cuando se listó "se casó conviviente" como un código para esta respuesta. Si un entrevistador hábil entendió en ambos casos la historia de la entrevistada, pudo haber codificado el cuestionario adecuadamente. Sin embargo, la secuencia y fraseología de las preguntas PECFAL propician mayor confusión y error en la codificación que las provenientes de la EMF.

Por último, en el cuestionario de la EMF se pregunta a las mujeres que se identifican a sí mismas como solteras, en el presente, "¿ha estado usted unida o casada alguna vez?", y si contestan afirmativamente se les pregunta, “entonces, ¿vive en unión libre o es separada, divorciada o viuda?". En cambio en el cuestionario PECFAL, a las mujeres solteras se les pregunta, " ¿ha estado usted alguna vez unida sin casarse?" (las cursivas son nuestras). De esta forma, en la encuesta PECFAL, a diferencia de la FMF, pudo haber sido posible que se omitieran las historias maritales de 
algunas mujeres que estuvieron casadas con anterioridad pero que se dijeron solteras en el momento de la entrevista. Sin embargo, es improbable que esta diferencia en el diseño del cuestionario haya tenido un efecto digno de atención en las diferencias de las tasas de legalización en cuestión.

Tomadas en conjunto, estas diferencias en el diseño de las dos encuestas nos dicen que: 1) en la encuesta PECFAL era menos probable que se registraran las uniones libres de corta duración que se legalizaron, con respecto a las uniones que permanecieron intactas o que resultaron en separación a la fecha de la entrevista, y 2) aunque se registrara la unión libre, el cuestionario PECFAL pudo haber codificado erróneamente la legalización como otro tipo de terminación. El cuadro 5 apoya esta última hipótesis en alguna medida: las probabilidades de separación y viudez de doble decremento son más elevadas para la muestra PECFAL que para la muestra comparable de la EMF. En virtud de que la mayoría de las diferencias se observan en el primer año de unión, las legalizaciones de uniones breves pueden haber sido incorrectamente registradas como separaciones. Debido a que el diseño del cuestionario de la EMF es más cuidadoso y menos ambiguo, y que además se introducen de manera frecuente preguntas de control en la historia de las uniones, podría ser que las estimaciones de legalización a partir de los datos de la EMF fuesen más confiables que las que se basan en los datos de la encuesta PECFAL.

\section{Variaciones en la probabilidad de legalización}

Como lo demuestra el cuadro 6, la probabilidad de legalización de las primeras uniones difiere sustancialmente de la de las segundas y sucesivas, para las entrevistadas por la EMF. Únicamente $19 \%$ de uniones de orden

CUADRO 5

Probabilidades acumuladas de decremento doble para legalización, separación y viudez (muestras comparables de la encuesta EMF y PECFAL)

\begin{tabular}{|c|c|c|c|c|}
\hline \multirow{2}{*}{$\begin{array}{l}\text { Duración de } \\
\text { de la unión } \\
\text { (años) }\end{array}$} & \multicolumn{2}{|c|}{ EMF } & \multicolumn{2}{|c|}{ PECFAL } \\
\hline & Legalización & $\begin{array}{c}\text { Separación } \\
\text { o viudez }\end{array}$ & Legalización & $\begin{array}{c}\text { Separación } \\
\text { o viudez }\end{array}$ \\
\hline 1 & .17 & .04 & .10 & .09 \\
\hline 2 & .21 & .07 & .13 & .12 \\
\hline 3 & .24 & .10 & .16 & .15 \\
\hline 5 & .28 & .14 & .18 & .20 \\
\hline 10 & .36 & .22 & .23 & .27 \\
\hline 15 & .45 & .27 & .28 & .32 \\
\hline \multicolumn{5}{|c|}{ Número inicial } \\
\hline de casos & & 776 & & 712 \\
\hline
\end{tabular}




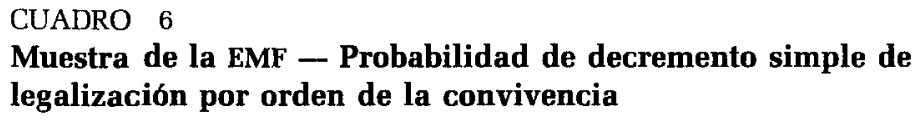

\begin{tabular}{lll}
\hline 1 & .20 & .03 \\
2 & .26 & .05 \\
3 & .30 & .06 \\
5 & .36 & .08 \\
10 & .46 & .19 \\
Número inicial & & \\
de casos & 1708 & 420
\end{tabular}

Fuente: Encuesta Mexicana de Fecundidad (1976).

superior estaban legalizadas después de 10 años de duración, comparado con $46 \%$ para las primeras uniones. Estos resultados sugieren que las mujeres con más de una unión prefieren la cohabitación al matrimonio, que los hombres están menos dispuestos a casarse con mujeres que hayan tenido otras parejas y que tal vez tengan niños, o que un buen número de convivencias de orden superior incluyen a mujeres casadas con anterioridad, que no pueden volver a casarse porque nunca se han divorciado formalmente. En virtud de las numerosas diferencias en la frecuencia de legalización por orden de unión y las cifras relativamente pequeñas de uniones de orden superior, restringimos el resto del análisis a las primeras convivencias.

Si bien los resultados del cuadro 2 indican que es más probable que las mujeres de cohortes más viejas, que eran mayores en el momento de la primer unión y que tenían una mayor escolaridad, tuvieran un matrimonio legal como primera unión, todavía debe determinarse si estas mismas caraterísticas aumentan la probabilidad de legalización para mujeres que inicialmente forman una convivencia. En el cuadro 7 presentamos las probabilidades de legalización por algunas duraciones de unión seleccionadas y conforme a la edad de la mujer en el momento de la entrevista, edad al inicio de la primera unión, y escolaridad. En nuestro trabajo anterior con la encuesta PECFAL, encontramos que en México era más probable que una mujer legalizara su convivencia si era católica creyente (Goldman y Pebley, 1981). Por desgracia en la EMF no existe ninguna información sobre religión.

Los resultados en el cuadro 7 sugieren que la legalización es más común entre las cohortes más jóvenes y entre las mujeres que se casan después de los 17 años. Existen diferencias sustanciales por escolaridad, pues las mujeres con más escolaridad tienen mayor probabilidad de legalizar una unión. Estos resultados son similares a los de la encuesta PECFAL si 
CUADRO 7

Probabilidad acumulada de legalización de decremento simple, por duración de la unión, edad a la entrevista, edad a la unión y escolaridad (exclusivamente para primeras uniones)

\begin{tabular}{|c|c|c|c|c|c|c|}
\hline \multirow{2}{*}{$\begin{array}{l}\text { Duración de } \\
\text { la unión } \\
\text { (años) }\end{array}$} & \multirow[b]{2}{*}{$<20$} & \multicolumn{3}{|c|}{ Edad a la entrevista } & \multicolumn{2}{|c|}{ Edad a la unión } \\
\hline & & $20-29$ & $30-29$ & $40-49$ & $\leqslant 17$ & $>17$ \\
\hline 1 & .22 & .22 & .21 & .17 & .19 & .23 \\
\hline 2 & .34 & .27 & .25 & .21 & .24 & .29 \\
\hline 3 & .37 & .33 & .28 & .26 & .28 & .34 \\
\hline 4 & $-{ }^{*}$ & .42 & .33 & .29 & .34 & .38 \\
\hline 10 & -* & .55 & .43 & .37 & .44 & .50 \\
\hline \multicolumn{7}{|l|}{$\begin{array}{l}\text { Número inicial } \\
\text { de casos }\end{array}$} \\
\hline \multirow{2}{*}{$\begin{array}{l}\text { Duración de } \\
\text { la unión } \\
\text { (años) }\end{array}$} & \multicolumn{6}{|c|}{ Educación } \\
\hline & Ninguna & Alguna & & Primaria & Secun & y más \\
\hline 1 & .17 & .22 & & .22 & & \\
\hline 2 & .22 & .28 & & .27 & & \\
\hline 3 & .25 & .32 & & .31 & & \\
\hline 5 & .30 & .39 & & .38 & & \\
\hline 10 & .38 & .51 & & .50 & & \\
\hline $\begin{array}{l}\text { Número inicial } \\
\text { de casos }\end{array}$ & 561 & 1147 & & 1022 & & \\
\hline
\end{tabular}

Fuente: Encuesta Mexicana de Fecundidad (1976)

* Menos de 20 casos.

bien las diferencias educacionales son más impresionantes para las entrevistadas de la EMF. Después de 5 años de duración, $22 \%$ de las entrevistadas sin escolaridad en la encuesta PECFAL habían legalizado su cohabitación, en comparación con $24 \%$ con alguna escolaridad; las cifras respectivas para la encuesta de la EMF son de 30 a 39 por ciento. El efecto más grande de la escolaridad que se derivó de la EMF puede ser resultado de la inclusión de más mujeres con escolaridad elevada en la última categoría.

En el cuadro 7 intentamos describir las variaciones en la legalización más que probar hipótesis sobre los determinantes de la legalización. El análisis de los determinantes tendría que ser multivariado, pues aspectos como la edad al inicio de la convivencia y el nivel de escolaridad pueden estar relacionados.

\section{Legalización y fecundidad}

Ya se mencionó que algunos demógrafos han hecho conjeturas sobre la posibilidad de que el mayor incentivo de las parejas que viven en unión 
libre al legalizar la unión es el deseo de legitimar a los hijos. Uno de los hallazgos más importantes en nuestros estudios sobre legalización en América Latina fue que el conocimiento de un embarazo o nacimiento de una criatura no aumentaba sustancialmente la frecuencia de legalización. Dadas las limitaciones de la muestra PECFAL, nuestro deseo era probar esta hipótesis utilizando una encuesta más reciente y representativa a nivel nacional, como lo es la EMF.

Es mucho más complicado probar la magnitud de la asociación entre fecundidad y legalización que otras comparaciones en este análisis. La simple comparación de tasas de legalización entre convivencias sin ningún embarazo o nacimientos, y convivencias con embarazos o nacimientos, arroja resultados absurdos. Las legalizaciones que se hacen poco después del inicio de la cohabitación, por lo general se llevan a cabo antes de que la mujer haya tenido oportunidad de embarazarse. De ahí que la primera categoría se caracterice por una probabilidad de legalización muy elevada a duraciones tempranas.

La comparación adecuada tendría que partir del cálculo de la exposi. ción para mujeres antes y después del inicio de un embarazo (o nacimiento). Una tabla de vida se basa en la exposición por duraciones desde el inicio de la cohabitación hasta el término de la convivencia (o fecha de entrevista) para mujeres sin embarazos dentro de la unión, combinada con la exposición por duraciones desde el inicio de la cohabitación hasta el inicio del embarazo, para el resto de las mujeres. La otra tabla de vida se basa en la exposición por duraciones desde el inicio del embarazo hasta el término de la unión (o fecha de la entrevista) para mujeres con un embarazo dentro de la unión. En ambas tablas de vida la duración se mide como tiempo desde el inicio de la cohabitación.

En el cuadro 8 se muestran algunos valores seleccionados que resultaron de las tablas de vida. Para el total de la muestra (que se observa en la primera columna) las mujeres tienen una probabilidad ligeramente mayor de legalizar la unión durante el primero o segundo año después del embarazo, que aquelias que no estaban embarazadas. Sin embargo, la probabilidad acumulada de legalización después de cinco años es un poco más elevada para las mujeres que han tenido un embarazo que para las que no lo han terido ( $38 \%$ frente a $30 \%$ ). El efecto de un embarazo, hasta donde exista, parece ser gradual más que inmediato y afecta sólo a las uniones que ocurrieron después de $1965 .^{9}$

\footnotetext{
${ }^{9}$ En la mayoría de las duraciones las diferencias entre las tasas de legalización ( $q_{x}$ ) para mujeres con y sin embarazo no sor: estadísticamente significativas. No obstante, la ausencia de diferencias significativas en algunas de las duraciones de orden superior puede deberse al tamaño tan reducido de la muestra en la categoría "sin embarazo".
} 
CUADRO 8

Probabilidad acumulada de legalización por duración, para uniones con o sin embarazo

\begin{tabular}{|c|c|c|c|c|c|}
\hline \multirow{2}{*}{$\begin{array}{l}\text { Duración de } \\
\text { la unión } \\
\text { (años) }\end{array}$} & \multirow[b]{2}{*}{ Total } & \multicolumn{4}{|c|}{ Fecha de la primera unión } \\
\hline & & Pre-1960 & $1960-64$ & $1965-69$ & $1970^{\star}$ \\
\hline \multicolumn{6}{|c|}{ Sin embarazo } \\
\hline 1 & .20 & .16 & .20 & .22 & .22 \\
\hline 2 & .24 & .22 & .22 & .24 & .28 \\
\hline 3 & .28 & .25 & $-{ }^{\star}$ & $-{ }^{\star}$ & $-{ }^{*}$ \\
\hline 5 & .30 & .27 & $-{ }^{*}$ & $-^{*}$ & $-{ }^{*}$ \\
\hline 10 & - $^{*}$ & - $^{\star}$ & $-^{\star}$ & $-^{\star}$ & - - \\
\hline \multicolumn{6}{|c|}{ Con embarazo } \\
\hline 1 & .22 & .16 & .18 & .27 & .27 \\
\hline 2 & .27 & .19 & .22 & .33 & .35 \\
\hline 3 & .32 & .23 & .27 & .38 & .39 \\
\hline 5 & .38 & .27 & .34 & .44 & .42 \\
\hline 10 & .44 & .36 & .43 & .47 & .47 \\
\hline Número inicial & & & & & \\
\hline de casos & 1707 & 583 & 266 & 315 & 543 \\
\hline
\end{tabular}

Fuente: Encuesta Mexicana de Fecundidad (1976)

* Menos de 20 casos.

\section{Estabilidad de uniones consensuales}

Los resultados de Goldman (1981) y de Potter y Ojeda (1982) indican que las parejas que viven en unión libre en América Latina tienen mayor probabilidad de separarse que las parejas unidas en matrimonio legal, aun cuando se tomen en cuenta la edad a la unión y las características sociales de las parejas. Potter y Ojeda (1982) utilizando la información de la EMF, señalan que "existe una fuerte suposición de que la decisión de legalizar una convivencia dependerá de la evaluación (tal vez principalmente de la mujer) de la estabilidad de la unión" (p. 2) ¿Es más probable que permanezcan unidas las parejas en convivencia legalizada que las parejas que se casan sin ningún periodo previo de cohabitación?

El cuadro 9 muestra una comparación, mediante el uso de tablas de vida, de las probabilidades de que un primer matrimonio termine en separación, con y sin cohabitación previa al matrimonio. Para los matrimonios que estaban precedidos de cohabitación, la duración se mide como tiempo desde la legalización. Nuestros resultados anteriores, derivados de las encuestas PECFAL (Goldman y Pebley, 1981) en los cuatro países que se incluyeron, indicaron que los matrimonios con cohabitación previa tenían menos probabilidades de disolución. Los resultados de la EMF, sin embargo, muestran que las probabilidades de disolución son iguales, sin importar si los matrimonios tuvieron o no cohabitación previa.

Una de las posibles razones de la ausencia de diferencias en las tasas 


\section{CUADRO 9}

\section{Probabilidad acumulada de separación para parejas con o sin cohabitación previa al matrimonio}

\begin{tabular}{lcc}
\hline $\begin{array}{l}\text { Duración del } \\
\text { matrimonio } \\
\text { (años) }\end{array}$ & $\begin{array}{c}\text { Sin cohabitación } \\
\text { previa }\end{array}$ & $\begin{array}{c}\text { Con cohabitación } \\
\text { previa }\end{array}$ \\
\hline 1 & .01 & .01 \\
2 & .02 & .02 \\
3 & .03 & .03 \\
5 & .04 & .04 \\
10 & .07 & .07 \\
Número inicial & & \\
de casos & 1575 & 238 \\
\hline
\end{tabular}

Fuente: Encuesta Mexicana de Fecundidad (1976).

de disolución entre matrimonios legalizados y matrimonios legales desde su inicio, es que existen dos procesos de selección en operación. Algunos estudiosos han sugerido que las parejas que piensan que tienen una relación más estable pueden formar un matrimonio legal desde el inicio, soslayando el periodo de prueba que proporciona una convivencia; entre las parejas que desde el inicio se deciden por un periodo de prueba únicamente se legalizan las uniones más estables (Quilodrán, 1980; Potter y Ojeda, 1982). De esta forma, los matrimonios legales parecen igualmente estables sin importar el proceso mediante el que se alcanzan.

\section{Resumen}

Hemos examinado en este documento la frecuencia y las variaciones de la legalización en México, utilizando la información de la Encuesta Mexicana de Fecundidad (EMF) 1976-1977. Nuestros resultados indican que a pesar de que la convivencia es bastante común entre las mujeres mexicanas, un buen número de convivencias se legalizan con posterioridad. Aproximadamente $30 \%$ de las primeras convivencias se legalizan dentro de un periodo de cinco años después de formadas. La vasta mayoría de convivencias ( $84 \%$ ) se disuelven dentro de un periodo de 25 años de duración, ya sea por legalización, separación o viudez. La frecuencia de legalización es similar en las áreas rurales y en las urbanas, si bien las convivencias son más comunes en las áreas rurales. Es más probable que se legalicen las primeras convivencias que las de orden subsiguiente. Es más probable que se casen con sus parejas las mujeres de cohortes más recientes, que empiezan una unión a edades más avanzadas y que tienen mayor escolaridad.

Se ha argumentado que el embarazo puede ser un factor importante que precipite la legalización de una convivencia. Nuestro análisis ante- 
rior, que se basa en la información de las encuestas PECFAL en áreas rurales en cuatro países de América Latina -en donde se incluye México-, indica que no había ninguna diferencia en la probabilidad de legalización en las uniones con o sin embarazo. Los resultados que se derivan del análisis de la información de la EMF, apoyan ampliamente este resultado de manera general. Sin embargo, parece que para las uniones que comenzaron después de 1965 existe una diferencia moderada después de los primeros años de unión.

Para terminar, consideramos la posibilidad de que los matrimonios que terminaban en legalización fuesen más estables que los primeros matrimonios de parejas que no habían tenido cohabitación previa al matrimonio. Nuestros resultados indican que no existe diferencia en la probabilidad de separación entre los dos grupos. Estos hallazgos contrastan con los resultados previos de la encuesta PECFAL, que indican que la cohabitación ejerce un efecto estabilizador en el matrimonio.

\section{Bibliografía}

Camisa, Zulma, "La nupcialidad de las mujeres solteras en América Latina”, en Notas de población, núm. 18, 1978, pp. 9-76.

Comisión Económica para América Latina [CEPAL], Población y desarrollo en América Latina, Fondo de Cultura Económica, México, 1975.

Flores, Carmen Elisa y Noreen Goldman, An Analysis of Nuptiality Data in the Colombia National Fertility Survey, Reporte Científico núm. 11, Encuesta Mundial de Población, 1980, Londres.

Goldman, Noreen, "Dissolution of First Unions in Colombia, Panama and Peru", en Demography, núm. 18, 1981, pp. 659-679.

Goldman, Noreen y Anne R. Pebley, "Consensual Unions in Latin America", en Social Biology, núm. 28 (1-2), 1981, pp. 49-61.

Michielutte, R., C.E. Vicent, C.M. Cochrane y C.A. Haney, "Consensual and Legal Marital Unions in Costa Rica", en International Journal of Comparative Sociology, núm. 14, 1973, pp. 119-128.

Mortara, G., Characteristics of the Demographic Structure of the American Countries, Secretariado General de la Unión Panamericana, Organización de los Estados Americanos, Washington, 1964.

Ordorica, Manuel y Joseph E. Potter, Evaluation of the Mexican Fertility Survey, 19761977, Reporte Científico núm. 21, Encuesta Mundial de Fecundidad, Londres, 1981.

Potter, Joseph E. y Norma Ojeda de la Peña, "Dissolution of First Unions in Mexico", documento presentado a la reunión anual de la Asociación de Población de América, San Diego, California, 1982.

Quilodrán, Julieta, "Tipos de uniones maritales en México", en Investigación demográfica en México-1980, Consejo Nacional de Ciencia y Tecnología, México, 1982.

Rosero, L.B., Nupcialidad y fecundidad en cuatro zonas rurales de América Latina, CELADE, Serie C, núm. 1008, San José, Costa Rica, 1978.

Secretaría de Programación y Presupuesto, Coordinación General del Sistema Nacional de Información, Encuesta Mexicana de Fecundidad: primer informe nacional, México, 1979.

Stycos, J.M., Human Fertility in Latin America, Universidad de Cornell, Ithaca, 1968.

Yaukey, D. y T. Thorsen: "Differential Female Age at Marriage in Six Latin American Cities", en Journal of Marriage and the Family, núm. 34, 1972, pp. 375-379. 